\title{
Optical Transducers Based on Amorphous Si/SiC Photodiodes
}

\author{
Manuela Vieira ${ }^{1,2,3}$, Paula Louro ${ }^{1,2}$, Miguel Fernandes ${ }^{1,2}$, Manuel A. Vieira ${ }^{1,2}$, \\ and João Costa ${ }^{1,2}$ \\ ${ }^{1}$ Electronics Telecommunications and Computer Dept., ISEL, Lisbon, Portugal \\ ${ }^{2}$ CTS-UNINOVA, Quinta da Torre, 2829-516, Caparica, Portugal \\ ${ }^{3}$ DEE-FCT-Universidade Nova de Lisboa, Quinta da Torre, 2829-516, Caparica, Portugal
}

\begin{abstract}
Amorphous $\mathrm{Si} / \mathrm{SiC}$ photodiodes working as photo-sensing or wavelength sensitive devices have been widely studied. In this paper single and stacked a-SiC:H p-i-n devices, in different geometries and configurations, are reviewed. Several readout techniques, depending on the desired applications (image sensor, color sensor, wavelength division multiplexer/demultiplexer device) are proposed. Physical models are presented and supported by electrical and numerical simulations of the output characteristics of the sensors.
\end{abstract}

Keywords: Amorphous Si/SiC photodiodes, photonic, optoelectronic, image sensors, demultiplexer devices, optical amplifiers.

\section{Introduction}

The Tunable optical filters are useful in situations requiring spectral analysis of an optical signal. A tunable optical device is a device for wavelength selection such as an add/drop multiplexer (ADM) which enables data to enter and leave an optical network bit stream without having to demultiplex the stream. They are often used in wavelength division multiplexing (WDM) systems [1]. WDM systems have to accomplish the transient color recognition of two or more input channels in addition to their capacity of combining them onto one output signal without losing any specificity (wavelength and bit rate) Only the visible spectrum can be applied when using polymer optical fiber (POF) for communication [2]. So, the demand of new optical processing devices is a request.

\section{Contribution to Sustainability}

These sensors are different from the other electrically scanned image sensors as they are based on only one sensing element with an opto-mechanical readout system. No pixel architecture is needed. The advantages of this approach are quite obvious like the feasibility of large area deposition and on different substrate materials (e.g., glass, polymer foil, etc.), the simplicity of the device and associated electronics, high resolution, uniformity of measurement along the sensor and the cost/simplicity of the detector. The design allows a continuous sensor without the need for pixel-level 
patterning, and so can take advantage of the amorphous silicon technology. It can also be integrated vertically, i. e. on top of a read-out electronic, which facilitates low cost large area detection systems where the signal processing can be performed by an ASIC chip underneath.

In this paper we present results on the optimization of different multilayered a-SiC: $\mathrm{H}$ heterostructures for spectral analysis in the visible spectrum. A theoretical analysis and an electrical simulation are performed to support the wavelength selective behavior.

\section{Device Configuration and Spectral Analysis}

The semiconductor sensor element is based on single or stacked a-SiC:H p-i-n structures using different architectures, as depicted in Fig. 1. All devices were produced by PE-CVD on a glass substrate. The simplest configuration is a $\mathrm{p}-\mathrm{i}-\mathrm{n}$ photodiode (NC11) where the active intrinsic layer is a double layered a-SiC:H/a- Si:H thin film. In the other the active device consists of a p-i'(a-SiC:H)-n / $\mathrm{p}-\mathrm{i}(\mathrm{a}-\mathrm{Si}: \mathrm{H})-\mathrm{n}$ heterostructures. To decrease the lateral currents, the doped layers (20 $\mathrm{nm}$ thick) of NC12 have low conductivities and are based on a-SiC:H [3].

Deposition conditions are described elsewhere

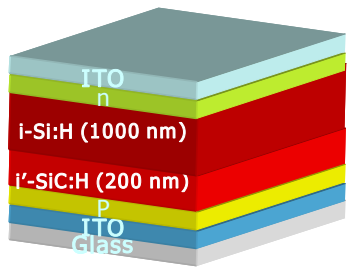
$[4,5]$. Full wavelength detection is achieved based on spatially separated absorption of different wavelengths. The blue sensitivity and the red transmittance were optimized, respectively, using a thin a-SiC:H front absorber $(200 \mathrm{~nm})$ with an optical gap of $2.1 \mathrm{eV}$ and a thick a-SiH back NC11 absorber $(1000 \mathrm{~nm})$ with an optical gap around 1.8

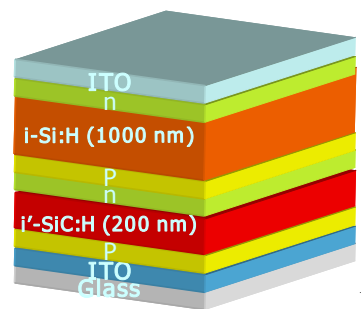
$\mathrm{eV}$. The thicknesses of both absorbers result from a trade-off between the full absorption of the blue light in the front diode and the green light across both. As a result, both front and back diodes act as optical filters confining, respectively, the blue and the red optical carriers, while the green ones are absorbed across both [6]. The devices were characterized through spectral response at $1 \mathrm{kHz}$ and photocurrent-voltage measurements. To test the sensitivity of the device under different electrical and optical bias three modulated monochromatic

Fig. 1. Device configuration

lights channels: red (R: $\left.626 \mathrm{~nm} ; 51 \mu \mathrm{W} / \mathrm{cm}^{2}\right)$, green (G: $\left.524 \mathrm{~nm} ; 73 \mu \mathrm{W} / \mathrm{cm}^{2}\right)$ and blue (B: $470 \mathrm{~nm} ; 115 \mu \mathrm{W} / \mathrm{cm}^{2}$ ) and their polychromatic combinations (multiplexed signal) illuminated separately the device. The generated photocurrent was measured under positive and negative voltages $(+1 \mathrm{~V}<\mathrm{V}<-10 \mathrm{~V})$, with steady state red $\left(\lambda_{\mathrm{R}}=626\right.$ $\left.\mathrm{nm} ; \Phi_{\mathrm{R}}=102 \mu \mathrm{W} / \mathrm{cm}^{2}\right)$, green $\left(\lambda_{\mathrm{G}=} 524 \mathrm{~nm} ; \Phi_{\mathrm{G}}=71 \mu \mathrm{W} / \mathrm{cm}^{2}\right)$ and blue $\left(\lambda_{\mathrm{B}=4} 470 \mathrm{~nm}\right.$; $\left.\Phi_{\mathrm{B}}=293 \mu \mathrm{W} / \mathrm{cm}^{2}\right)$ optical bias and without it $\left(\Phi_{\mathrm{R}, \mathrm{G}, \mathrm{B}}=0\right)$. The semiconductor sensor element is based on single or stacked a-SiC:H p-i-n structures using different architectures, as depicted in Fig. 1. 
In Fig. 2, for NC11 and NC12 it is displayed the spectral photocurrent under different applied voltages.
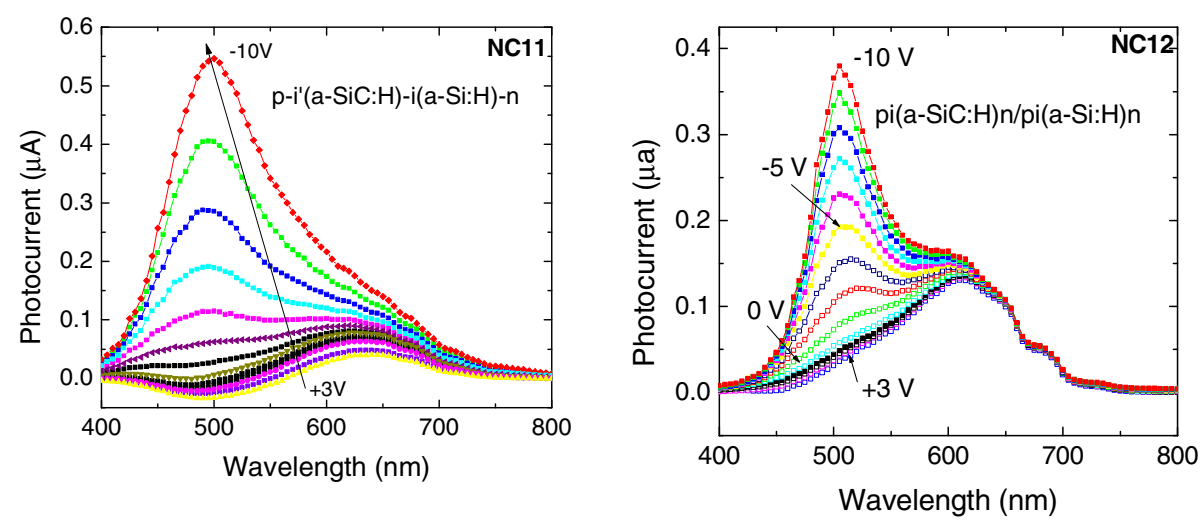

Fig. 2. Spectral response under different applied bias for three different architectures

Data show different behaviors when single and double structures are compared. In the single configuration the electrical field is asymmetrically distributed across the graded i'i -layer leading to a collection efficiency that is only dependent on the light depth penetration across it. In the double, the contribution of both front and back diodes are evident and the effect of the internal p-n junction crucial on the device functioning and future applications.

\section{Wavelength (De)Multiplexer Device}

In optical communications different wavelengths (color channels) which are jointly transmitted must be separated (demultiplexed) to decode the multiplexed information.

Fig. 3 displays, under positive and negative bias, the multiplexed signals, acquired with NC12 device, due to the simultaneous transmission of three independent bit sequences, each one assigned to one of the red (R: $626 \mathrm{~nm})$, green $(\mathrm{G}: 524 \mathrm{~nm})$ and blue (B: 470nm) color channels. At the top of the figure, the optical signal used to transmit the information is displayed to guide the eyes on the different ON-OFF states.

To recover the transmitted information ( 8 bit per wavelength channel, 2000bps) the multiplexed signal, during a complete cycle $(0<\mathrm{t}<\mathrm{T})$, was divided into eight time slots, each corresponding to one bit where the independent optical signals can be ON (1) or OFF (0). As, under forward bias, the device has no sensitivity to the blue channel (Fig. 2), the red and green transmitted information can be identified from the multiplexed signal at $+1 \mathrm{~V}$. The highest level corresponds to both channels ON (R\&G: $\mathrm{R}=1, \mathrm{G}=1)$, and the lowest to the OFF-OFF stage $(\mathrm{R}=0 ; \mathrm{G}=0)$. The two levels inbetween are related with the presence of only one channel $\mathrm{ON}$, the red $(\mathrm{R}=1, \mathrm{G}=0)$ or the green $(R=0, G=1)$ (see horizontal labels in Fig. 3). To distinguish between these two situations and to decode the blue channel, the correspondent sub-levels, under 
reverse bias, have to be analyzed. From Fig. $2 b$, it is observed that the green channel is more sensitive to changes on the applied voltage than the red, and that the blue only appears under reverse bias. So, the highest increase at $-8 \mathrm{~V}$ corresponds to the blue channel $\mathrm{ON}(\mathrm{B}=1)$, the lowest to the $\mathrm{ON}$ stage of the red channel $(\mathrm{R}=1)$ and the intermediate one to the $\mathrm{ON}$ stage of the green $(\mathrm{G}=1)$ (vertical labels in Fig. 3). Using this simple algorithm and MATLAB as programming environment, the independent red, green and blue bit sequences can be decoded, in real time, as: R [00011011], $\mathrm{G}$ [001101010] and B [00101011] in agreement with the optical signals used to transmit the information.

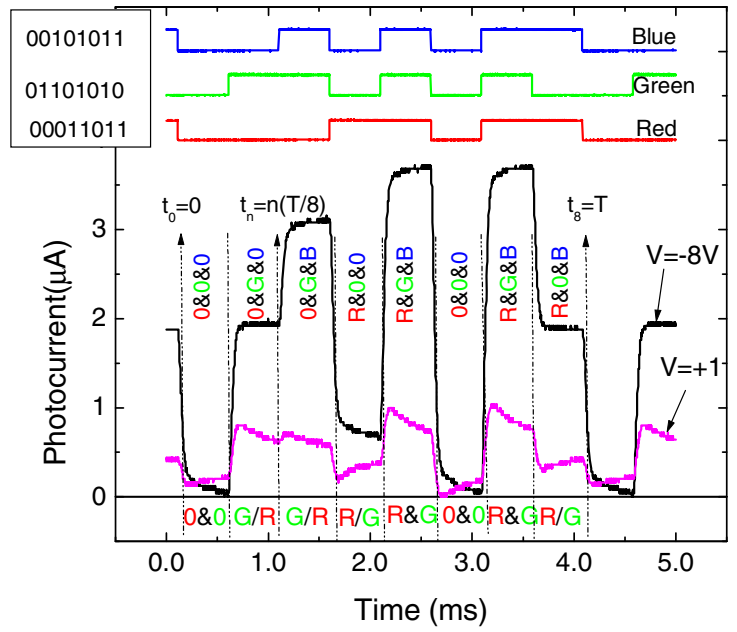

Fig. 3. Multiplexed signals under reverse and forward bias. On the top, the optical signal used to transmit the information guide the eyes on the different ON-OFF states. The recovered bit sequences are shown as an insert.

\section{Optical Bias Controlled Wavelength Discrimination}

In Fig. 4a the spectral photocurrent at different applied voltages is displayed without and under red, green and blue background irradiation. In Fig. $4 \mathrm{~b}$ the ratio between the spectral photocurrents under red, green and blue steady state illumination and without it (dark) are plotted.

When an external electrical bias (forward or reverse) is applied to a double pin structure, it mainly influences the field distribution within the less photo excited subcell: the back under blue irradiation and the front under red steady bias [8]. Under negative bias the blue bias enhances the spectral sensitivity in the long wavelength ranges and quenches in the short wavelength range. The red bias has an opposite behavior; it reduces the collection in red/green wavelength ranges and amplifies the blue one. The green optical bias only reduces the spectral greenish photocurrent keeping the other two almost unchangeable. 

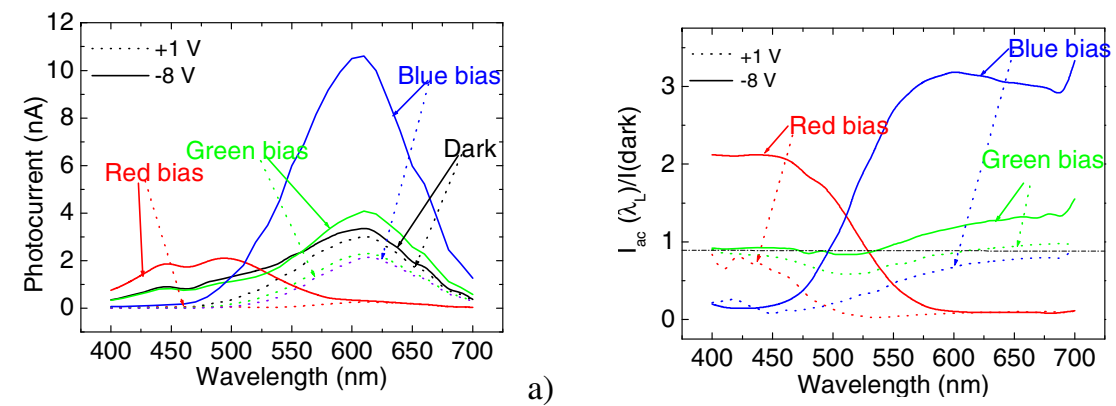

b)

Fig. 4. a) Spectral photocurrent @ $+1 \mathrm{~V},-8 \mathrm{~V}$ without (dark) and under red, green and blue optical bias. b) Ratio between the photocurrents under red, green and blue steady state illumination and without it (dark).
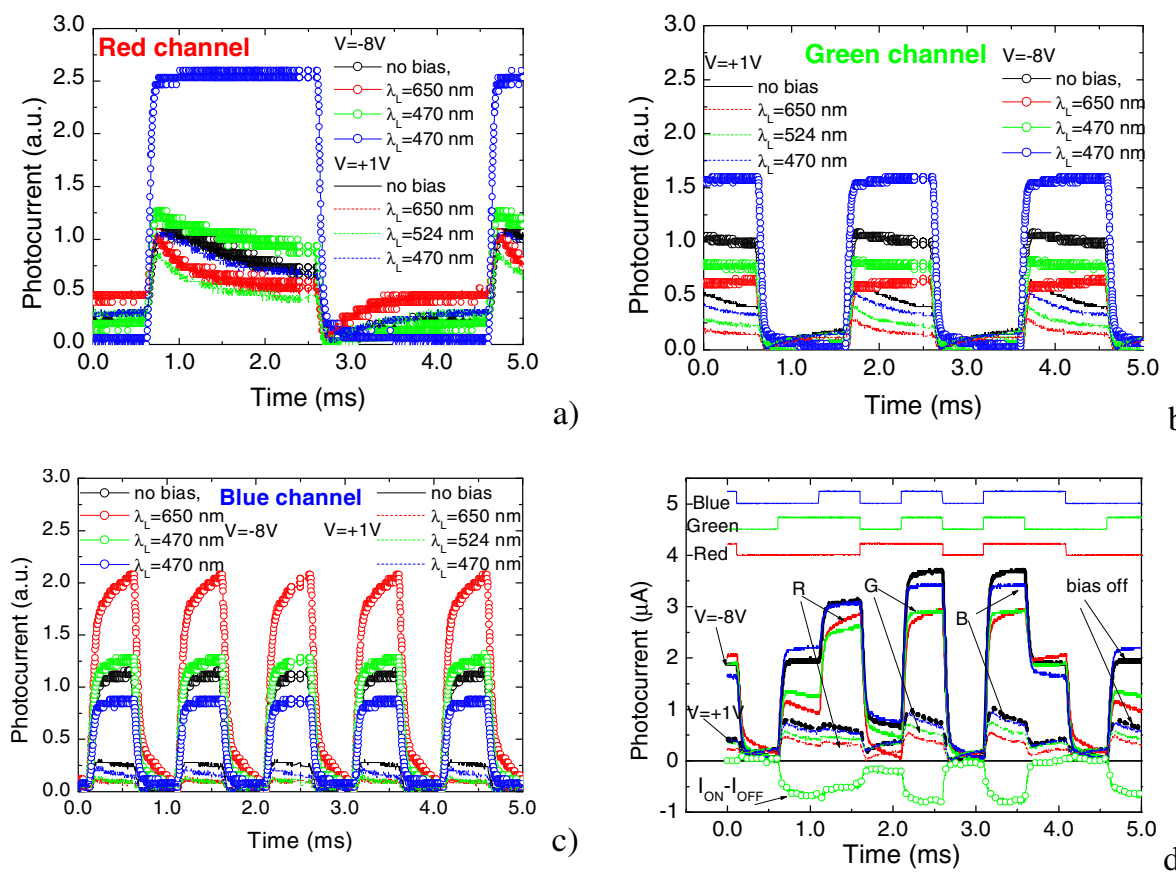

b)

Fig. 5. Input red (a) green (b) and blue (c) signals under negative and positive bias without and with red, green and blue steady state optical bias. d) Multiplexed signals @ -8V/+1V (solid /dot lines); without (bias off) and with (R, G, B) green optical bias.

To analyze the self bias amplification under transient conditions and uniform irradiation, three monochromatic pulsed lights $(\mathrm{R}, \mathrm{G}$ and $\mathrm{B}$ input channels illuminated separately $\mathrm{NC} 12$ device. Steady state red, green and blue optical bias was superimposed separately and the photocurrent generated measured at $-8 \mathrm{~V}$ and $+1 \mathrm{~V}$. 
In Fig. 5a, Fig. 5b and Fig. 5c the signal is displayed for each monochromatic channel separately and in Fig. 5d for the bit sequence shown on the top of figure.

Results show that when an optical bias is applied it mainly enhances the field distribution within the less photo excited sub-cell. Even at high frequencies the blue irradiation amplifies the red $\left(\alpha_{R}=2.25\right)$ and the green $\left(\alpha_{G}=1.5\right)$ channels and quenches the blue one $\left(\alpha_{B}=0.8\right)$. The red bias has an opposite behavior, it reduces the red and green channels and amplifies de blue $\left(\alpha_{R}=0.9, \alpha_{G}=0.5, \alpha_{B}=2.0\right)$. The green optical bias reduces the green channel $\left(\alpha_{\mathrm{G}}=0.75\right)$ keeping the other two almost unchangeable. This optical nonlinearity makes the transducer attractive for optical communications and can be used to distinguish a wavelength, to read a color image, to amplify or to suppress a color channel or to multiplex or demultiplex an optical signal. In Fig. 5c the green channel is tuned through the difference between the multiplexed signal with and without green optical bias

\section{Electrical Model}

The silicon-carbon pi'npin device is a monolithic double pin photodiode structure with two red and blue optical connections for light triggering (Figure 6a). Based on the experimental results and device configuration an electrical model was developed [7]. Operation is explained in terms of the compound connected phototransistor equivalent model displayed in Figure 6b.

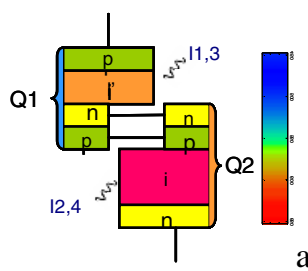

a)
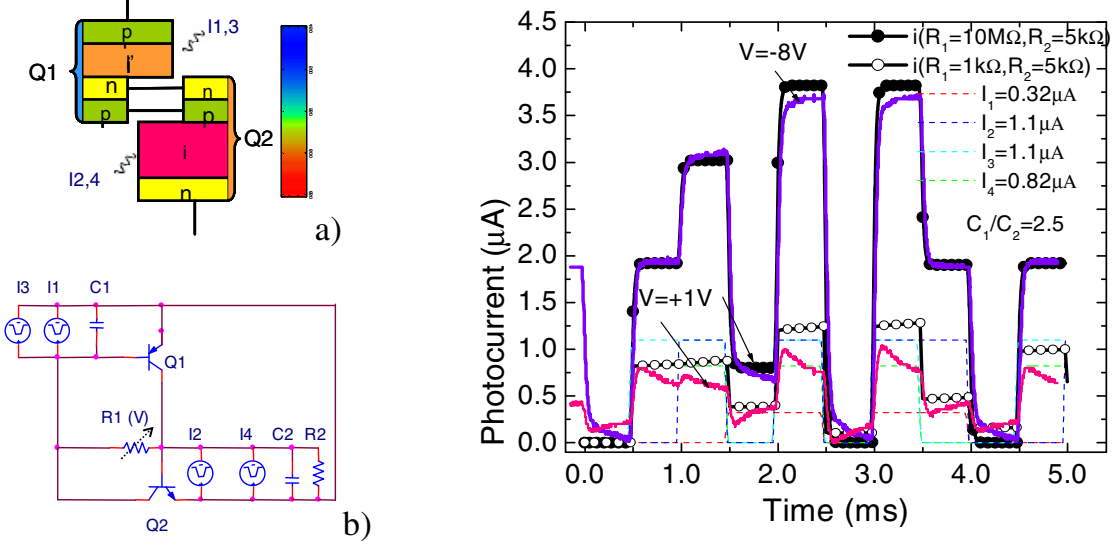

Fig. 6. a) Compound connected phototransistor equivalent model. b) $a c$ equivalent circuit. c) Multiplexed simulated (symbols) and experimental (solid lines) results under positive and negative dc bias. The current sources used as input channels (dash lines) are displayed.

The capacitive effects due to the transient nature of the input signals are simulated through $\mathrm{C}_{1}$ and $\mathrm{C}_{2}$ capacitors. $\mathrm{R}_{1}$ and $\mathrm{R}_{2}$ model the dynamic resistance of the internal and back junctions, respectively. The photocurrent under positive (open symbols) and negative (solid symbols) $d c$ bias is displayed in Figure 6c. We have used as input parameters the experimental values of Figure 3. The input transient current sources used to simulate the photons absorbed in the front (blue, $\mathrm{I}_{1}$ ), back (red, $\mathrm{I}_{2}$ ), or across 
both (green, $\mathrm{I}_{3}$ and $\mathrm{I}_{4}$ ) photodiodes are also displayed (dash lines). To validate the model the experimental multiplexed signals at $-8 \mathrm{~V}$ and $+1 \mathrm{~V}$ are also shown (lines).

Good agreement between experimental and simulated data was observed. The expected levels, under reversed bias, and their reduction under forward bias are clearly seen (Figure 3). If not triggered $\mathrm{ON}$ by light the device is nonconducting (low levels), when turned $\mathrm{ON}$ it conducts through different paths depending on the applied voltage (negative or positive) and trigger connection $\left(\mathrm{Q}_{1} \mathrm{Q}_{2}\right.$ or both).

Under negative bias (low $\mathrm{R}_{1}$ ) the base emitter junction of both transistors are inversely polarized and conceived as phototransistors, thus taking advantage of the amplifier action of neighboring collector junctions, which are polarized directly. This results in a charging current gain proportional to the ratio between both collector currents $\left(\mathrm{C}_{1} / \mathrm{C}_{2}\right)$. The device behaves like a transmission system able to store, amplify and transport all the minority carriers generated by the current pulses, through the capacitors $\mathrm{C}_{1}$ and $\mathrm{C}_{2}$. Under positive bias (high $\mathrm{R}_{1}$ ) the device remains in its non conducting state unless a light pulse $\left(\mathrm{I}_{2}\right.$ or $\left.\mathrm{I}_{2}+\mathrm{I}_{4}\right)$ is applied to the base of $\mathrm{Q}_{2}$. This pulse causes $\mathrm{Q}_{2}$ to conduct because the reversed biased n-p internal junction behaves like a capacitor inducing a charging current across $\mathrm{R}_{2}$. No amplification effect was detected since $\mathrm{Q}_{1}$ acts as a load and no charges are transferred between $\mathrm{C}_{1}$ and $\mathrm{C}_{2}$.

The optical amplification under transient condition also explains the use of the same device configuration in the Laser Scanned Photodiode (LSP) image and color sensor [9]. Here, if a low power monochromatic scanner is used to readout the generated carriers the transducer recognize a color pattern projected on it acting as a color and image sensor. Scan speeds up to $10^{4}$ lines per second are achieved without degradation in the resolution.

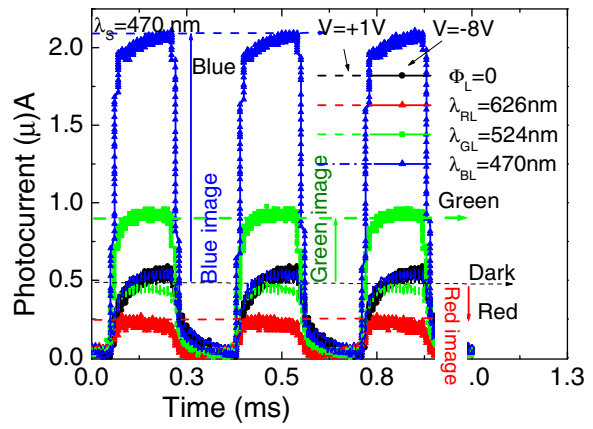

Fig. 7. LSP color sensor

For the color image sensor only the red channel is used (Fig. 6, $\mathrm{I}_{2} \neq 0$, $\mathrm{I}_{1}=\mathrm{I}_{3}=\mathrm{I}_{4}=0$ ). To simulate a color image at the XY position, using the multiplexing technique, a low intensity moving red pulse scanner $\left(\Phi_{S}, \lambda_{S}\right)$, impinges in the device in dark or under different red, green and blue optical bias (color pattern, $\left.\Phi_{\mathrm{L}}, \lambda_{\mathrm{RGB}, \mathrm{L}}, \Phi_{\mathrm{L}}>\Phi_{\mathrm{S}}\right)$. Fig. 7 displays the experimental acquired electrical signals. The image signal is defined as the difference between the photocurrent with (light pattern) and without (dark) optical

bias. Without optical bias $\left(\Phi_{\mathrm{L}}=0\right)$ and during the red pulse, only the minority carriers generated at the base of $\mathrm{Q}_{2}$ by the scanner, flow across the circuit $\left(\mathrm{I}_{2}\right)$ either in reverse or forward bias. Under red irradiation (red pattern, $\Phi \neq 0, \lambda_{\mathrm{RL}}$ ) the base-emitter junction of $Q_{2}$ is forward bias, the recombination increases reducing $I_{2}$ thus, a negative image is observed whatever the applied voltage. Under blue $\left(\Phi \neq 0, \lambda_{\mathrm{BL}}\right)$ or green $\left(\Phi \neq 0, \lambda_{\mathrm{BL}}\right)$ patterns irradiations the signal depends on the applied voltage and consequently, on $\mathrm{R}_{1}$. Under negative bias an optical enhancement is observed due to the amplifier action of adjacent collector junctions which are always polarized directly. Under positive bias the device remains in its non conducting state, unless the 
red pulse ( $\mathrm{I}_{2}$, dark level) is applied to the base of $\mathrm{Q}_{2}$. No amplification occurs and the red channel is strongly reduced when compared with its value under negative voltage. Under blue irradiation, the internal junction becomes reverse biased at $+1 \mathrm{~V}$ (blue threshold) allowing the blue recognition. The behavior under a green pattern depends on the balance between the green absorption into the front and back diodes that determines the amount of charges stored in both capacitors. Under negative bias both the green component absorbed either in the front (blue-like) or at the back (red-like) diodes reaches the output terminal while for voltages at which the internal junction n-p becomes reversed (green threshold), the blue-like component is blocked and the red-like reduced. So, by using a thin a-SiC:H front absorber optimized for blue collection and red transmittance and a back a-Si:H absorber to spatially decouple the green/red absorption, the model explains why a moving red scanner (probe beam) can be used to readout RGB the full range of colors at each location without the use of a pixel architecture.

\section{Conclusions}

Different architectures based on silicon carbon pin devices were studied both theoretically and experimentally. Results show that when the doped layers has low conductivities and are based on a-SiC:H material the devices are optical and voltage controlled and can be used as optical filter, amplifier or multiplexer /demultiplexer devices in the visible range.

\section{References}

1. Bas, M.: Fiber Optics Handbook, Fiber, Devices and Systems for Optical Communication, ch. 13. McGraw-Hill, Inc., New York (2002)

2. Randel, S., Koonen, A.M.J., Lee, S.C.J., Breyer, F., Garcia Larrode, M., Yang, J., Ng'Oma, A., Rijckenberg, G.J., Boom, H.P.A.: Advanced modulation techniques for polymer optical fiber transmission. In: Proc. ECOC 2007 (Th 4.1.4), Berlin, Germany, pp. 1-4 (2007)

3. Vieira, M., Fernandes, M., Louro, P., Fantoni, A., Vygranenko, Y., Lavareda, G., Nunes de Carvalho, C.: Mat. Res. Soc. Symp. Proc., vol. 862, pp. A13.4 (2005)

4. Vieira, M., Fantoni, A., Fernandes, M., Louro, P., Lavareda, G., Carvalho, C.N.: Thin Solid Films 515(19), 7566-7570 (2007)

5. Vygranenko, Y., Wang, K., Vieira, M., Sazonov, A., Nathan, A.: Appl. Phys. Lett. 95, 263-505 (2009)

6. Louro, P., Vieira, M., Vygranenko, Y., Fantoni, A., Fernandes, M., Lavareda, G., Carvalho, N.: Mat. Res. Soc. Symp. Proc., vol. 989, p. A12.04 (2007)

7. Vieira, M.A., Vieira, M., Fernandes, M., Fantoni, A., Louro, P., Barata, M.: MRS Proceedings Amorphous and Polycrystalline Thin-Film Silicon Science and Technology 2009, vol. 1153, p. A08-0 (2009)

8. Vieira, M., Fantoni, A., Louro, P., Fernandes, M., Schwarz, R., Lavareda, G., Carvalho, C.N.: Vacuum 82(12), 1512-1516 (2008)

9. Vieira, M., Fantoni, A., Fernandes, M., Louro, P., Rodrigues, I.: Mat. Res. Soc. Symp. Proc.762@2003 A.18.13 